\title{
CBT in Affective Disorders - New Frontiers
}

\author{
Larissa Wolkenstein and Martin Hautzinger
}

Additional information is available at the end of the chapter

http://dx.doi.org/10.5772/54236

\section{Introduction}

The basic idea of Cognitive Behavioral Therapy (CBT) for depression (as well as for other disorders) is the intercorrelation of mood, thinking and behavior [1]. These three modalities are reciprocally correlated. That means that a negative mood can result in a reduced level of activity and a negative and constricted way of thinking but at the same time specific behaviors may lead to a bad mood and a negative way of thinking or one thought (or a bad memory) may narrow the behavior and depress mood.

The first step of a successful CBT is to build up a good therapeutic relationship. In a second step, psychoeducation is followed by concrete therapy goals formulation. Within the next CBT sessions one of the central challenges for the therapist is to deduce the basic rationale of intercorrelation of mood, behavior and cognition from the personal experiences described by the patient and to illustrate how this interrelation may end up in a negative spiral. Thus, a rationale of depression has to be provided and the problems of the patient have to be related to this rationale. The main goal of CBT for depression is to stop the negative spiral and to initiate constructive changes on the behavioral as well as on the cognitive level. Thus, CBT undermines cognitive and behavioral maintenance factors of depression leading to reevaluations of underlying assumptions and beliefs so as to reduce vulnerability and risk factors [2].

A first step to cause changes on the cognitive level, i.e. to reach cognitive restructuring, is to identify negative automatic thoughts the patient has in his/her everyday life as well as the cognitive biases that underlie these negative automatic thoughts. This may be for example 'all-or-nothing thinking' or 'emotional reasoning'. Within the CBT, cognitions are defined as hypotheses instead of being defined as facts and the patient is inspired to test these hypotheses. For this purpose therapists use the technique of Socratic dialogue. In a Socratic dialogue open questions are used to point out inconsistencies in the conclusions of the patient without devaluating, or showing up the patient. Questions therapists pose are short and concise, refer to proximate statements of the patient and are helpful to develop new 
insights. If a cognition or thought has proven to be wrong alternative explanations and reattributions are developed together with the patient. Pros and Cons as well as short- and long-term consequences of specific attitudes are considered and weighed up.

Besides these techniques that intend to reach cognitive restructuring, behavioral components are also part of the CBT for depression. These components aim to influence patient's behavior directly, to provide immediate help, quick changes and facilitations, to increase positive experiences, as well as to overcome behavioral deficits. The main goals of the behavioral therapeutic elements are 1. to breach the vicious circle of inactivity, passivity, social withdrawal and depressive symptoms, 2. to structure everyday life and the course of specific actions and behaviors, 3. to interrupt and detract from rumination, 4 . to acquire new skills and resources to reduce depressive behaviors and relapses, and 5. to support the cognitive interventions by the use of role plays, the enhancement of positive activities, and behavioral exercises that can be understood as experiments and reality testing [1].

One of the first behavioral therapeutic elements that are used in the therapy of depression is the enhancement of positive activities alongside a reduction of negative activities as this allows for early positive experiences and positive reinforcements in the context of the therapy. Often, weekly activity schedules are used for this purpose, so that patients can record their activities. To vividly illustrate the association between behavior and mood patients are often asked to also record how they feel after the different activities they display. On this background the motivation to include more positive activities into the weekly activity schedule can be build up.

Another behavioral therapeutic component is the improvement of social competences. The number of social contacts of depressed patients is often low and social competences of the patients are impaired. Consequently, social relationships of depressed patients are often not satisfactory. In many cases it does not suffice just to reduce the depressive symptomatology to overcome the reduced social contacts or the deficient social competences of the patients. Therefore, the improvement of social competences, communication skills and problem solving skills is an essential part of a promising CBT. The basic instruments in this context are the role play, behavioral exercises and the inclusion of partners and family members.

Within the last therapy sessions of a CBT it is important to address the issue of relapse and recurrence of depression. After CBT, patients should be enabled to get along with future depressive symptoms, crises and possible relapses. Therefore, patients need to repeat and practice what they have learned thoroughly so as to be able to use all the techniques on their own after the CBT has been finished. Probable crises should be brought up to reflect possibilities to get along self-directed. Therefore, all materials that have been used in the therapy should be handed out to the patient so that he/she can use them as utilities. Furthermore, to prevent relapses it has proven to be helpful to fade out therapeutic contacts slowly by extending the time interval between the therapy sessions. This enables the therapist to be available for at least one year in case of crises or relapses after the main part of the therapy has been finished. Many patients value the offering of the so-called boostersessions. 


\section{Effectiveness and efficacy of cognitive behavioral therapy in the treatment of depression}

Placebo-controlled studies that compared the efficacy of cognitive behavior therapy (CBT) with that of antidepressant medication (ADM) found out that CBT is as efficacious as ADM in the treatment of patients with mild to severe forms of depression and that both treatments are superior to placebos [3-5]. Spoken numerically, about $40-60 \%$ of the patients respond to a CBT treatment [6]. However, early studies concluded that ADM is superior to pill-placebo in the treatment of severe depressions whereas CBT is not [7]. Even though this conclusion was mainly based on one study it had a great impact on the perception of treatment effectiveness and resulted in suggestions from the American Psychiatric Association that ADM should be preferred over CBT for patients with more severe forms of depression [8]. Yet, subsequently conducted studies found out that this conclusion was wrong and that CBT fares as well as ADM with severely depressed patients [4, 9]. Accordingly, ADM should not be considered to be superior to CBT for the acute treatment of severe forms of depression. However, it seems as if the efficacy of CBT in the treatment of severe forms of depression depends on a high level of therapist experience $[4,10]$.

Going beyond the immediate effects of ADM and CBT it has to be kept in mind that while medication usually has a rapid and robust effect it has been shown to prevent the return of depressive symptoms only for as long as it is taken [8]. Thus, the intake of medication does not seem to alter the risk factors that lead to a subsequent recurrence. For that reason medication does not reduce the risk of relapse once its use is terminated [11]. That is why most patients with chronic or recurrent forms of depression are encouraged to stay on medication for an indefinite period [6]. In contrast, CBT appears to have an enduring effect that reduces risk of relapse not only during the time of therapy but also after treatment termination [11-12]. In a meta-analysis of Gloaguen and colleagues it has for example been shown that the one-year relapse rate for patients previously treated with CBT was approximately $25 \%$, whereas $50 \%$ of patients previously treated with ADM relapsed in the same period. Furthermore, it has been shown that the preventative effect of prior CBT is at least as large in magnitude as that effect that is produced by keeping patients on medication [13-14]. A combination of CBT and medication holds the benefits of each and thus may enhance the probability of treatment response over a monotherapy, especially in chronic depressions [11].

Despite lower relapse rates associated with CBT as compared to medication relapse is still a substantial problem in CBT of depression. In contrast to addictive disorders where the focus has already many years ago been laid on relapse prevention research has not paid a lot of attention to relapse-prevention in depressive disorders. Patients who have recovered from recurrent major depressive disorder face an $80 \%$ rate of recurrence if they do not get further prophylactic treatment [15]. To handle and reduce that risk physicians prescribe either continuation- or maintenance-phase medication. In contrast, it still doesn't seem to be custom to offer psychotherapeutic relapse-prevention after patients responded to the acute phase of depression-specific psychotherapeutic treatments. One concrete suggestion for 
improving the long-term outcome of depressive patients after a CBT comes from Jarrett and colleagues who suggest to include a continuation or maintenance phase into the CBT [16]. Over an 8-month period the authors found that a CBT including a continuation phase (C$\mathrm{CT}$ ) reduced relapse estimates significantly more than a control condition that was defined as evaluation without CT (10\% vs. 31\%). Over a 2-year follow-up, also including a CBT-free period, C-CT significantly reduced relapse and recurrence estimates in patients with earlyonset depression (16\% vs. $67 \%$ in control). Furthermore, if patients had unstable remission during the late phase of acute treatment, C-CT also significantly reduced relapse and recurrence estimates to $37 \%$ as compared to $62 \%$ in the control group. Thus, the authors conclude that risk factors influence the necessity for relapse-prevention. In the 12-month follow-up of another study that has been conducted by DeRubeis and colleagues it has been found that patients who completed the CBT and had up to three optional booster sessions afterwards were significantly less likely to relapse than patients who switched to placebo after the acute phase CBT $(31 \%$ vs. $76 \%)$ [4, 13]. Another promising way to reduce relapserates seems to be mindfulness-based cognitive therapy (MBCT) that is designed to train recovered patients with recurrent depression to disengage from depressogenic thinking that may mediate relapse. Teasdale and colleagues reported that recovered patients who received treatment as usual (TAU) and MBCT instead of TAU alone had significantly lower relapse rates - however this was only the case for patients with three or more previous depressive episodes [17].

CBT is not only effective in the treatment of acute depression and in relapse-prevention but also in the treatment of residual depressive symptoms. The largest study to date that addressed this issue included 158 patients with residual symptoms after a recent depressive episode that has been treated with ADM [18-19]. These patients either received clinical management alone or clinical management plus cognitive therapy over twenty weeks with two subsequent booster sessions. Patients received continuation and maintenance medication. CBT reduced relapse rates ( $29 \%$ vs. $47 \%$ in the clinical management group) as well as persistent residual symptoms. CBT also increased full remission rates at 20 weeks. Furthermore, the addition of CBT produced improvements in social and psychological functioning.

In contrast to CBT there is only little knowledge concerning the long-term efficacy of other psychosocial treatments in relation to that of CBT. One study that aimed to test the theory of change that underlies CBT undertook a component analysis of CBT [20] and thereby treated patients either exclusively with behavioral activation (BA), a combination of BA and teaching of skills to modify automatic thoughts (AT), or the full CBT treatment also including the components that focus on core schema. Jacobson and colleagues [20] did not find an evidence that the complete CBT was superior to either component treatment neither at the termination of acute treatment nor at the 6-month follow-up. Moreover, both BA as well as AT also altered negative thinking and dysfunctional attributional styles of the patients - just as did the complete CBT treatment. Another study even demonstrated that an expanded BA model was as effective as was ADM with respect to the reduction of acute distress of depressed patients independently of the level of initial depression severity, and 
superior to CBT when regarding more severely depressed patients [21]. In a more recent randomized controlled trial of interpersonal psychotherapy (IPT) versus CBT it has been shown that even though patients in a treatment as usual condition improve just as do patients in a CBT or an IPT condition, IPT and CBT seem to be superior to TAU in at least some analyses [22]. Another study that aimed to compare the efficacy of interpersonal psychotherapy (IPT) and CBT demonstrated that both therapies are effective in treating depressive disorders, but that CBT seems to be superior in treating severe depression [23]. In contrast, de Mello and colleagues who conducted a systematic review of research findings on the efficacy of IPT for depressive disorders came to the conclusion that CBT is less efficacious than IPT [24].

When searching for the effect size associated with CBT for depression the picture gets less homogenous compared to the question if CBT has an effect at all. CBT has been reported to have a medium effect size $(d=.67)$ relative to a number of different control conditions as for example non-specific controls or the absence of treatment [25]. As expected the effect size of CBT is larger when it is compared to waiting list controls $(\mathrm{d}=0.88)$ than when it is compared to treatment as usual $(\mathrm{d}=0.38)$ or non-specific controls $(\mathrm{d}=0.38)$ [25]. Moreover, a combination of CBT with ADM seems to be superior to ADM alone as it is associated with a somewhat larger effect size $(\mathrm{d}=0.27)$. In an older meta-analysis Cuijpers, van Straten, Andersson and van Oppen [26] included 53 studies in which seven types of psychotherapy have been compared with other psychological treatment to examine the efficacy of psychotherapies for depression in adults. The authors concluded that all forms of psychotherapy were efficacious in treating depression. However, it seemed as if IPT was somewhat more efficacious $(\mathrm{d}=0.20)$ whereas non-directive supportive treatment was somewhat less efficacious $(d=-0.13)$ than the other treatments. Furthermore, it has been reported that the efficacy of group CBT $(d=0.15)$ does not significantly differ from the efficacy of individual CBT [27]. However, it has to be mentioned that the quality of the studies that have been included in the respective meta-analysis was rather low. In a more recent meta-analysis Cuijpers and collaborators [28] found evidence that the effects of psychotherapy for depression have been overestimated in former meta-analyses and that the quality of the studies examining psychotherapy for depression is associated with the effect sizes that have been found. Accordingly, they reported a standardized mean effect size of $d=0.22$ for high quality studies as compared to a mean effect size of $d=0.74$ in lower-quality studies, even after controlling for the control condition used. This difference has been significant. That lower study quality seems to be related with larger effect sizes has also been confirmed by a more recent review [29]. Moreover, Cuijpers and collaborators [30] found that in dependence of the kind of instrument used to measure improvement following a psychotherapy the effect sizes of studies examining the effect of psychotherapy for depression differ significantly so as to clinician-ratings are associated with a significantly greater effect size than are self-report measures. This has to be kept in mind when drawing conclusions from efficacy-studies.

Besides the efficacy of CBT in sense of recovery and relapse-prevention that is at least comparable with or even superior to the efficacy of ADM it is important to mention that 
CBT is also more cost-effective than ADM in the longer term [31]. And not only the efficacy of individual CBT has been well-documented but there are also hints that group CBT seems to be efficacious. In this context Brown and colleagues [32] compared the clinical effectiveness, costs, treatment preference, attrition and patient satisfaction of group and individual CBT in a naturalistic clinical study. Despite the fact that patients preferred individual CBT at baseline, they found no significant differences between group and individual CBT in depressive and distress symptoms at post-treatment and at 3-month follow-up. Furthermore, there were no differences in attrition and satisfaction between the two groups. However, the cost-effectiveness was higher for group CBT. Thus, if this finding holds true in further studies, it could be considered more frequently to conduct group CBT instead of individual CBT.

\section{Mediators and moderators of treatment effects of CBT for depression}

During the last years the search for moderators and mediators of the response to cognitive behavioral treatments for depression has been intensified. The reason therefore is that not only the response rate could be increased but also the costs could be decreased if we knew which patients are likely to respond to a CBT treatment and which processes mediate the treatment response. A moderator can be understood as a patient characteristic that is present before the treatment and independent of treatment assignment. Furthermore, it has an interactive effect with the treatment condition on the treatment outcome. Thus, a moderator variable influences the direction or the magnitude of the relationship between the independent (treatment condition) and the dependent (outcome) variable. It is important to distinguish between moderator variables that predict differential response to treatment and prognostic factors that predict the outcome in general and irrespective of treatment assignment [33]. In contrast, a mediator variable is an intervening variable that accounts for the relationship between the independent and the dependent variable. Thus, a mediator variable changes due to the treatment and in turn correlates with treatment outcome [33]. In the following section we would like to highlight the main findings concerning moderator and mediator variables of CBT for depression (without drawing a claim on completeness as there are several studies in this field even though the number is small as compared to studies examining moderators and mediators of ADM).

While during the last years the search for possible moderators of the response to CBT treatment for depression has especially focused on the neurobiological activity pattern of the patients, it has already taken other variables into account long before.

Some patient characteristics with unclear status: Prognostic factors or moderator variables for the response to CBT for depression?

An early study of Sotsky et al. [34] in which 239 patients with a depressive disorder have been included has highlighted that six patient characteristics as well as the severity of the depression patients reported before the beginning of the treatment predict treatment outcome (not only of CBT but also of IPT, ADM with clinical management, and placebo with clinical management). These characteristics comprised social dysfunction, cognitive dysfunction, 
expectation of improvement, endogenous depression, double depression and the duration of the current episode. Partly, these findings have been confirmed by Carter and colleagues who also found that a treatment response to either CBT or IPT is predicted by the perceived logicalness of the therapy (which conforms to the predictive value of the afore mentioned expectation of improvement), recurrent depression, and childhood reasons for the depression [35]. Furthermore, Fournier and colleagues found that older age and chronic depression predict relatively poor outcome following CBT as well as following ADM [36]. Uncontrolled pre-post-studies revealed that being married and displaying a lower initial symptom severity are prognostic of better response to CBT [37-38], however, it is unclear whether this finding is treatment-specific. Furthermore, patients who are above average intelligent seem to be more suitable for CBT than patients with lower intelligence [36, 39].

In the last decades it has also been paid attention to the role of negative cognitive schemata in moderating the effect of CBT. Thereby, it has been assumed that having particular negative cognitive schemata leads to severe and chronic problems that are not changeable with standard CBT and therefore require specific schema-focused procedures [40]. Halford and colleagues examined if this hypothesis holds true for patients with depression or anxiety disorder and found that contrary to this assumption a greater initial endorsement of maladaptive schemata did not predict poor therapy response [41]. Again, it is unclear to date whether the endorsement of maladaptive schemata would also be associated with good treatment response to other psychosocial interventions. Thus, it remains unclear if this is a prognostic factor rather than a moderator variable.

Another variable that has repeatedly been examined is the tendency to ruminate or to distract in response to depressed mood. In this context Teismann and colleagues found that even after controlling for depression scores pretreatment greater symptom- and self-focused rumination pretreatment predicts more self- and interviewer-rated depression as well as greater general symptom severity and lower therapists' appraisal of success posttreatment [42]. However, in another study the same authors found that ruminative responses to depressed mood are neither predictive of the therapeutic alliance nor of patients' receptivity to therapeutic interventions as perceived by the therapist [43]. Moreover, while the pretreatment tendency to distract was not associated with posttreatment depression after a CBT another study found that it is predictive of the patients' receptivity in therapy and the goodness of the therapeutic alliance $[42,44]$. Again, it is not clear whether these findings are specific to CBT or also hold true for any other psychosocial treatment.

As it has been described before, during a CBT patients are taught to interrupt automatic emotional processing (e.g., rumination) with controlled processing (e.g., cognitive reappraisal). Accordingly, what a CBT seems to directly address is the sustained emotional reactivity that has repeatedly been associated with depression [45]. Furthermore, it has been shown that depression is associated with an increased and sustained reactivity in brain regions responsible for emotional processing, particularly the amygdala [46-48]. On this background Siegle, Carter and Thase [49] developed the idea that there might be an association between the neural correlates of sustained emotional processing pretreatment 
and the posttreatment recovery. To test for this hypothesis, the authors subjected their participants to undergo an fMRI during an emotional information processing task before they received a CBT. The authors highlighted that a stronger recovery following a CBT was associated with a decreased reactivity to negative words in the subgenual cingulate cortex and an increased reactivity in the amygdala - both pretreatment. The authors suppose that the pattern of decreased reactivity in the subgenual cingulate cortex in combination with the sustained amygdala reactivity to emotional stimuli reflects a ruminative coping style. Indeed, the patients' percent change in sustained amygdala activity was correlated with the self-reported tendency of the patients to ruminate, i.e., to think repetitively about their depressive symptoms. Accordingly, the authors suggest that their findings demonstrate that those patients benefit most from CBT who show an increased emotional reactivity pretreatment and who are not able to engage regulatory structures pretreatment. According to Siegle, Carter and Thase the latter has been illustrated by the decreased reactivity in the subgenual cingulated cortex pretreatment which suggests a deficient regulation. So, the authors conclude that the presence of emotion regulation deficits, which are targeted in $\mathrm{CBT}$, may be the key to recovery with this specific intervention.

\section{Findings concerning more "severe and complicated patients"}

There are some preliminary indications that especially in the treatment of more severe and complicated patients a greater expertise of therapists is required to reach a successful outcome. In this context it has been shown that among patients with more severe depression, who are functionally impaired and have primary support group problems, both BA as well as ADM seem to be superior to pill-placebo whereas CT does not [21]. Furthermore, in another study the personality disorder status of the patients included led to differential response: In this study CT was superior to ADM for patients without personality disorder whereas the reverse was true for patients with personality disorder. However, despite the poor response of patients with comorbid Axis II disorders to CT, nearly all patients who did respond to CT within this study sustained their response over a one-year follow-up [50]. On the background of these studies Coffman and colleagues [51] concluded that "there might be a subset of patients who see themselves as doing better with sustained attention to behavior change in time-limited treatment". However, as the authors correctly indicate, before drawing firm conclusion, this pattern of self-reported non-response to CT should be replicated. Interestingly, other studies that did only focus on increased comorbid personality disorder symptoms instead of diagnosed Axis II disorders found a reverse pattern: For example Carter and colleagues found that increasing comorbid personality disorder symptoms where associated with decreases in response to IPT but not to CBT [35]. Other studies have shown that CBT seems to be more effective for patients who display an elevated level of avoidant personality disorder symptoms whereas IPT seems to be superior for patients displaying an elevated level of obsessive-compulsive personality disorder symptoms [52]. Facing these rather heterogeneous findings concerning the influence of Axis II symptoms and Axis II disorder status on the response to CBT and other psychosocial treatments it gets clear that future investigations are needed to elucidate if this really can be understood as a moderator variable and if so if it is associated with higher or lower response rates to $\mathrm{CBT}$. 
In a more recent, prospective, randomized, observer blinded multicenter study Stangier and colleagues compared the rates of relapse in 180 completely remitted depressed patients with three or more previous episodes who either received TAU plus Maintenance Cognitive Therapy (MCT) or TAU plus Manualized Psychoeducation (MAPE) for eight months [53]. Afterwards the patients were followed up for one year. The authors found that time to relapse of MDE did not differ significantly between the two treatment conditions. However, there was a significant interaction between treatment condition and the number of previous MDE episodes ( $<5$ vs. $\geq 5$ ), indicating that in patients with 5 or more previous episodes MCT was significantly superior to MAPE, whereas this was not true for patients with less than 5 previous episodes. The authors conclude that CBT has significant effects on relapse prevention only in patients with a high risk of depression recurrence whereas for patients with a lower risk of recurrence, non-specific and structured education may be equally effective.

\section{Some moderating patient characteristics}

Some patient characteristics examined in the early Sotsky study [34] have been predictive for differential treatment outcome. For instance, a low social dysfunction predicted superior response to IPT while a low cognitive dysfunction predicted superior response to CBT and to ADM. In addition, high depression severity and impairment of function have been associated with a superior response to ADM and IPT while high work dysfunction predicted superior response only to ADM.

Fournier and colleagues found that marriage, unemployment, and having experienced a greater number of recent life events predict superior response to CBT as compared to ADM [36]. Interestingly, in another study Barber and Muenz found that married patients did better with CBT than they did with IPT whereas unmarried patients did better with IPT than with CBT [52].

Patient's "attachment avoidance" has been shown to be associated with a better response to CBT as compared to IPT [54]. Keeping in mind that CBT does not target close relationships as specifically as does IPT this result seems to be intuitive because one would expect that CBT should be less threatening to individuals with high attachment avoidance as compared to IPT. Furthermore, it has been reported that while avoidant and schizoid symptoms predicted poorer response to IPT this was not true for CBT [55].

Another variable that was assumed to interact with treatment group to differentially affect treatment outcome is the patients' preference concerning the intervention [56]. Kocsis and colleagues found that in a randomized multicenter trial that compared the efficacy of Cognitive Behavioral Analysis System of Psychotherapy (CBASP) with that of nefazodone or the combination of both for chronic depression there was an interactive effect of the treatment preference of the patients (as indicated at study entry) and treatment group on the outcome. Patients who preferred medication at study entry had a higher remission rate and a lower symptom severity at study exit if they received medication than if they received CBT. Similarly, patients who preferred CBASP had a higher remission rate and a lower symptom severity at study exit if they received CBASP than if they received medication. 
Thus, it can be concluded that patients' preference is a significant moderator of treatment response for chronically depressed patients. However, Leykin and colleagues, who also compared treatment outcomes of patients who received their preferred treatment via randomization versus those who did not, did not find a significant difference in the magnitude of symptom reduction between these two groups [57]. As did Kocsis and colleagues, Leykin and colleagues compared ADM with CBT - however, they did not specifically focus on chronically depressed patients.

\section{The mediating role of neural mechanisms}

Hence, as a primary goal of CBT is the replacement of automatic emotional reactivity with more controlled processing it can be assumed that on the neural level CBT might be accompanied by an increase of inhibitory executive control that helps to dampen automatic reactions that are reflected in an increased limbic activity. Indeed, recent studies have shown that CBT affects clinical recovery by modulating the functioning of specific sites in cortical and limbic regions. For example Goldapple and colleagues [58] conducted a resting state positron emission tomography (PET) study and found that treatment response following a CBT is associated with increases in the metabolic activity of the hippocampus, and the dorsal cingulate cortex alongside decreases in the metabolism of the dorsal, ventral, and medial frontal cortex. This study, regarded on the background of older studies that examined the effects of ADM [59-60], suggests that while ADM effects may be targeted to limbic regions and then forwarded CBT may rather target the prefrontal cortex by downtuning cognitive processes that take place automatically. This is also assumed by DeRubeis, Siegle and Hollon [61] in a comprehensive review of treatment outcomes and neural mechanisms of CT and ADM for depression. Furthermore, in a randomized controlled trial Kennedy and colleagues [62] have shown that a treatment response to CBT as well as a treatment response to ADM (venlafaxine) are associated with decreased glucose metabolism bilaterally in the orbitofrontal cortex and left medial prefrontal cortex alongside an increase in the metabolism of the right occipital-temporal cortex. However, metabolic changes in the anterior and posterior parts of the subgenual cingulate cortex and the caudate differentiated CBT and venlafaxine responders. Thus, the authors conclude that while response to either treatment was associated with a reduced metabolism in several prefrontal regions, only response to CBT seems to be associated with a reciprocal modulation of cortical-limbic connectivity.

Looking at further studies using functional neuroimaging techniques it gets obvious that different mediating brain mechanisms have been suggested and underpinned by single studies. One prospective study that included 16 medication-free patients suffering from an acute major depressive disorder has been conducted by Fu and colleagues [63]. During an fMRI scan pre- and posttreatment subjects performed an affect recognition task with morphed facial stimuli displaying sadness. In between patients received 16 CBT sessions. Pretreatment it has been shown that during the acute phase patients show an elevated amygdala-hippocampal activity as compared to healthy controls. At the end of the treatment the amygdala activity of the CBT responders has decreased to healthy niveau. Furthermore, the clinical response of the patients was significantly correlated with their 
baseline dorsal anterior cingulate activity. Thus, the authors suggest that anterior cingulate activity may be a predictor of treatment response to CBT. In a more recent study Ritchey and colleagues also used fMRI to assess neural responses to negative, neutral, and positive pictures in healthy controls and unmedicated, depressed patients - the latter pre- and posttreatment [64]. As did the study described before, the authors of this study also found evidence that the neural differences between depressed patients and healthy controls during emotion processing can be successfully addressed by CBT. Prior to the treatment the authors found that depressed patients show a reduced activity in the ventromedial prefrontal cortex, a diminished discrimination between emotional and neutral stimuli in the amygdala, caudate and hippocampus and an increased reactivity to negative versus positive pictures in the left anterior temporal lobe and the right dorsolateral prefrontal cortex. After the CBT patients exhibited activity increases in the ventromedial prefrontal cortex, an enhanced discrimination of emotional and neutral items in the amygdala and the caudate, as well as greater activity in response to positive versus neutral items in the left anterior temporal lobe. In accordance with the afore mentioned assumptions these neural changes may reflect increased engagement of processes that are involved in modulating responses to emotional stimuli. Furthermore, some of the identified pre-treatment differences between the two groups were predictive of the patients' treatment response. These included the hyperactivity in the ventromedial prefrontal cortex as well as the negativity bias in the left anterior temporal lobe and in the right dorsolateral prefrontal cortex.

CBT involves at least 3 broad treatment goals:

i. challenge patients' current way of coping with stressful events and problematic interpersonal relationship, attempts to enhance problem-solving capacities.

Neuropsychological studies show that working memory, cognitive flexibility and other executive funtioning processes are involved. This brings the dorsolateral prefrontal cortex (dlPFC) and the hippocampus $(\mathrm{Hc})$ into play and makes them potential targets of CBT.

ii. modify patients' perspectives about themselves and their relationships. Neuroimaging studies suggest that cortical midline structure, such as the ventral (vACC) and dorsal (dACC) anterior cingulate cortex, the ventralmedial (vmPFC) and dorsomedial $(\mathrm{dmPFC})$ prefrontal cortex, the posterior cingulate cortex $(\mathrm{pCC})$ are involved in self-related information processing. These brain areas are potential targets of CBT.

iii. assist patients in regulating distressing emotional states. CBT may impact brain functions associated with emotional processing, such as insular cortex (vlns), the amygdala (Am), the ventral and dorsal anterior cingulate cortex (vACC, dACC), the dorsolateral prefrontal cortex (dIPFC), the ventro-medial prefrontal cortex (vmPFC).

Table 1. Mechanisms of change - mediators of outcome 


\section{The mediating role of cognitive changes}

The assumption of cognitive theory is that depressive symptoms change while the patients change their beliefs and the way they process information [65]. Indeed, besides neural changes that seem to reflect an enhanced cognitive control over emotions after CBT, it has been shown that the habitual tendency to ruminate decreases over the course of a CBT - a finding that fits well to the results concerning the neural level [66]. However, this was nothing unique to CBT but has also been found to occur during a Mindfulness-Based Cognitive Therapy (MBCT) [66]. Moreover, it has been shown that during CBT as well as during ADM automatic negative thoughts decrease and this reduction in turn has been associated with overall clinical improvement, so as to patients who did not benefit from either intervention showed significantly less change on cognitive measures [67-68]. Further studies have confirmed that reductions in negative cognitions are not only present in CBT but that different CBT treatment components are comparably effective in reducing negative cognitions. For instance, cognitive therapy and behavioral activation produce similar reductions in negative cognitions [20,69]. According to Simons and colleagues these results suggests that cognitive change can be understood as a part of improvement rather than as the primary cause of improvement [68].To clarify the direction of effects DeRubeis and colleagues obtained depression severity scores as well as different measures of cognition not only in the beginning and in the end of a CBT and an ADM but also repeatedly during treatment admission [70]. The authors found that while cognitive change from pretreatment to midtreatment predicted change in depression from midtreatment to posttreatment in the CBT group, this was not the case in the ADM group. Thus, the authors concluded that cognitive changes indeed play a mediating role in CBT but not in ADM. Further support for the importance of cognitive changes in CBT comes from a more recent study in which it has been shown that among those patients who responded to a CBT both the development of CBT coping skills as well as in-session evidence of the independent use of these skills predicted a lower risk of relapse in the year following treatment [71]. Thus, it can be concluded that the development and use of CBT coping skills play an important role in relapse prevention. Similarly, Zindel Segal and colleagues [72] examined whether moodlinked changes in dysfunctional thinking following a sad mood induction predict relapse in depressed patients in remission. Therefore, patients were first randomly assigned to either ADM or CBT. Afterwards, patients who achieved remission underwent a sad mood induction and were then observed and repeatedly examined in the following 18 months. The authors reported two main findings: First, patients who recovered through ADM showed greater cognitive reactivity in response to a negative mood induction than those who recovered through CBT. Second, the magnitude of cognitive reactivity associated with the negative mood induction significantly predicted relapse over the subsequent 18 months - independently of the type of prior treatment.

Whereas changes in more superficial cognitions (i.e., automatic thoughts) seems to be a result of different interventions, changes in more basic cognitions, for example attributional style or dysfunctional beliefs, seem to be significantly larger and thus more unique to CBT [73]. Furthermore, it has been demonstrated that a change of these more stable information- 
processing proclivities does mediate the smaller rate of relapse following a CBT [73-74]. Thereby it proved to be important that patients do become less extreme in their informationprocessing instead of becoming unrealistically positive as the latter is also associated with a greater risk to relapse [74-75].

Another study that examined the mediating role of cognitive changes in the efficacy of CBT highlighted that many patients experience so-called "sudden gains" during a cognitive behavioral intervention [76]. Sudden gains refer to large symptom improvements in a single between-session interval that account for about fifty percent of these patients total improvement. These sudden gains were associated with a lower depression-level posttreatment and at an 18-month follow-up. Tang and DeRubeis [76] found that these sudden gains were preceded by considerable cognitive changes in the therapy session that has taken place immediately before the sudden gains and presume that these changes triggered the sudden gains. Furthermore, an improvement of the therapeutic alliance as well as additional cognitive changes followed in the therapy session immediately after the sudden gains. So, it can be assumed that considerable cognitive changes during one CBT session may lead to sudden gains that can be understood as the beginning of an upward spiral. Interestingly, one variable that also seems to predict later treatment outcome is the early improvement in the course of a CBT [77].

\section{New developments in the CBT for depression}

Within the last years there have been made some attempts to increase the access to CBT resulting in new types of CBT treatments as for example online- and telephone based treatments. Given the high stigmatization that is associated with psychiatric disorders and the therapeutic interventions for these disorders on the one hand and the increasing use of the internet and online programs on the other hand this development seems nothing but logical. Besides the easy accessibility and the potential to offer an approach in a nonstigmatizing environment there are some further advantages in conducting computerized CBT (cCBT). For instance, cCBT has the capacity to deliver structured input consistently with precision and is associated with comparably low costs [78]. Moreover, it has been shown that discontinuation of treatment is a problem that seems to be associated with faceto-face therapy [79]. According to some estimations about $47 \%$ of patients may drop out of psychotherapy [80]. Besides the already mentioned stigmatization limited time and the travel that is required to see a therapist might be barriers to the face-to-face treatment.

Before we summarize the findings concerning the efficacy of $\mathrm{CCBT}$ we would like to mention that computers and the internet can not only be used as an alternative to the traditional form of CBT but also as an adjunct to the individual contact with a therapist [78]: For example a computer can be used as a tool for screening or diagnosis and information can be provided either in written or in audio/video formats. Furthermore, structured selfhelp programs can be provided online whereby situations can be easily simulated and graded exposure in highly controlled conditions is possible. Besides that the patient and the therapist may communicate via emails, chats or forums either for therapeutic or 
administrative purposes. So, it can be concluded that the use of computers and the internet has the potential to enrich the therapeutic possibilities of the traditional face-to-face communication considerably.

However, what do we know concerning these new developments and especially concerning their efficacy? There are many cCBT programs developed for the treatment of depression named for example 'Colour Your Life' or 'Beating the Blues' and it has been shown that they are effective in the treatment of depression [81]. Foroushani and colleagues conducted a meta-review in which they included twelve systematic reviews covering results concerning the effectiveness of cCBT for mild to moderate depression [78]. This meta-review clarifies that while for single cCBT programs, as for example for 'ODIN', there is not enough data with good quality to conclude that they are really effective other cCBTs for depression, as for example 'MoodGYM', 'Beating the Blues', and 'Colour Your Life', have proven to be effective in reducing symptoms of depression. Looking at effect sizes that are reported for cCBT there are greater differences. Concerning to a review by Griffiths, Farrer and Christensten it ranges over different studies between 0.42 to 0.65 for CCBT interventions that included clinically depressed patients [81]. It has been reported that some cCBT packages seem to be more effective than treatment as usual (TAU), equally or less effective than bibliotherapy and no less effective than therapist-led CBT [78]. Even self-help internet interventions have been shown to reduce symptoms although this effect was smaller for depression than for anxiety disorders [78]. Furthermore, studies have indicated that it doesn't seem to be necessary that CCBT interventions are supported by mental health professionals [81] even though there is evidence that the provision of therapist support or the ongoing contact with users during an intervention seems to increase the effect of cCBT [82-83]. Even though it can be said that these first results seem to be quite promising the evidence indicating the effectiveness of CCBT is still limited so far and further randomized controlled trials are required. Another finding in the context of CCBT is that, as one would expect, CCBT reduces therapist time. However, to date it cannot be concluded that CCBT has superior effectiveness as compared to other forms of CBT because the therapist time of an intervention is only one variable amongst a number of variables that determine the costeffectiveness.

Besides cCBT CBT offered through the telephone (tCBT) has also been shown to have positive effects on depression [84]. Moreover, in a randomized controlled trial Mohr and colleagues compared a 16-week tCBT program with 16 weeks of a supportive emotionfocused therapy also administered via telephone and found that improvements over treatment were significantly greater for tCBT than for the telephone-administered supportive intervention [85]. Furthermore, the treatment gains were still present during 12month follow-up even though the differences between the two treatments were no longer evident. This study highlights that there is a specific effect of telephone-administered CBT that goes beyond the nonspecific effects of a supportive telephone-based intervention. In a more recent study it has been examined how tCBT performs as compared to face-to-face CBT with respect to attrition and symptom reduction [86]. Thereby, it has been shown that while both treatments were associated with a significant symptom reduction posttreatment, 
with CBT not being noteworthy superior to tCBT, more patients completed tCBT as compared to traditional CBT. However, even though all participants remained significantly less depressed at 6-month follow-up as compared to baseline participants receiving traditional CBT were significantly less depressed than participants receiving tCBT. Thus, the authors conclude that even though tCBT improves adherence compared with traditional CBT this might go at the cost of some increased risk of poorer maintenance of gains after the time of treatment. Further findings suggest that a telephone-administered CBT might not only be helpful as stand-alone therapy but also as an adjunct to pharmacotherapy. In a randomized trial with 393 depressed patients Ludman and colleagues evaluated usual care versus tCBT plus care management for primary care patients who were beginning an antidepressant treatment and highlighted that a brief, structured, telephone-administered CBT program can improve clinical outcomes for the majority of patients beginning antidepressant treatment in primary care [87].

So far there is only very little knowledge concerning moderator variables of cCBT. One study that investigated whether factors that are prognostic of depression outcome generally do also predict response to online CBT in a sample of depressed patients has been conducted by Button and colleagues [88]. In this study it has been shown that marital status as well as pretreatment severity predicted treatment response of the patients. Thereby, patients who were separated, widowed, or divorced showed a greater symptom reduction following the online CBT than did married patients. Having in mind earlier studies that repeatedly showed that in face-to-face CBT married patients have a better prognosis than unmarried individuals [37-38] this finding may indicate that online CBT is particularly beneficial for separated, widowed, and divorced patients and thus be a first hint on a moderating variable. However, this finding definitely requires replication as it is based on only one single study so far. Another finding of this study was that patients who were more severely depressed also displayed a superior treatment-derived benefit - a result that has already been reported in an earlier study concerning therapist-delivered internet psychotherapy for depression [89]. Furthermore, the authors found weak evidence that treatment effectiveness diminished with an increasing number of recent adverse life events. In contrast, age, educational level, and history of depression did not predict treatment response. Another variable that was assumed to possibly have the potential to moderate the effect of a cCBT and that has also been examined is the chronicity of depression [90]. However, no significant difference between chronic patients and non-chronic patients has been found in treatment outcome. In another randomized trial three hundred and three depressed patients were randomly assigned to either an unsupported online cCBT, a treatment as usual (TAU), or the combination of CCBT and TAU and demographic, clinical, cognitive, and short-term improvement variables have been assessed as potential moderator variables [91]. It could be shown that patients with higher levels of extreme (positive) responding as assessed by the Dysfunctional Attitude Scale-Form A benefited more from CCBT as compared to TAU, whereas patients who had a parental psychiatric history or the diagnosis of a major depressive disorder showed a better treatment response in CCBT and TAU as compared to TAU alone. Irrespective of treatment type current employment, low 
pretreatment illness severity as well as short-term improvement on clinical variables predicted a greater treatment-benefit.

Concerning mediating mechanisms of change in CCBT there are even les studies than concerning possible moderator variables. One study that evaluated treatment specificity and potential mediators of two online therapies for depressive symptoms included 263 depressed patients who were randomly assigned to either online CBT, online problemsolving Therapy (PST) or a waiting list control group [92]. Interestingly, no differing mechanisms of change have been found: Dysfunctional attitudes, worrying, a negative problem orientation, and perceived control all played a mediating role in online CBT as well as in online PST. Hence, the authors conclude that irrespective of the theoretical background of the therapy the psychological processes that lead to symptom reduction seem to be comparable.

\section{Main components of the cognitive behavioral therapy for bipolar disorders}

In contrast to unipolar depressive disorders bipolar disorders have long been regarded as purely biological-psychiatric disorders that can only be treated with medication. Therefore, psychotherapy has only had a supporting role, thought to be only helpful in encouraging medication adherence [93]. Due to the insight in the fundamental role of psychosocial factors in the onset and course of bipolar disorders [94-95] a more biopsychosocial explanatory model has only developed during the last decades and has brought about cognitive behavioral treatment approaches among other psychosocial interventions [96-98].

As in the treatment of other psychiatric disorders the first step of a successful CBT for bipolar disorders is the development of a good and sustainable therapeutic relationship. On the basis of this relationship psychoeducational sessions can be offered and concrete and achievable therapy goals can be formulated with the patient. Within the psychoeducation the therapist and the patient develop knowledge concerning factors that initiate and maintain bipolar symptomatology by building on the individual experiences of the patient. Thus, the role of genetic, biological, psychological and social factors in the development and maintenance of the illness are discussed and a vulnerability-stress-model is developed. Furthermore, the patient and the therapist deduce the intercorrelation of mood, behavior and thinking from the individual experiences of the patient so that a rationale of the bipolar disorder as well as a treatment rationale can be developed. The main goal of the CBT for bipolar disorders is to stop the downward spiral leading to depressive episodes as well as to stop the upward spiral leading to (hypo-)manic episodes. Therefore, within the psychoeducational sessions the symptomatology that is specific for depressive or (hypo-) manic episodes respectively is discussed. Furthermore, therapists focus on the course and prognosis of the illness, the role of risk factors and protective factors, as well as individual warning signs and prodromal symptoms. Moreover, medical and psychotherapeutic treatment opportunities are discussed. 
A further essential element of the CBT for bipolar disorders is a detailed analysis of the behavior and the preconditions that might have led to former affective episodes. On the basis of this knowledge potential triggers (that might be either negative or positive), early warning symptoms and prodromal symptoms as well as successful and unsuccessful coping strategies can be identified.

On this background it can be made out which further contents should be focused in the remaining therapy sessions. Thereby it might for example be useful to focus on the structure of the everyday life of the patient: While during depressive episodes many patients tend to withdraw and to reduce their daily activities to a minimum, during (hypo-)manic episodes many patients tend to overload their schedule excessively. It may also be useful to concentrate on problem solving or social competence skills, or on dysfunctional attitudes. Working on dysfunctional attitudes also comprises looking at cognitive errors that are characteristic for either depression (as for example all-or-nothing-thinking, overgeneralization, catastrophizing or mental filters) or (hypo-)mania (as for example positive fortune-telling, overevaluation of immediate satisfaction or the misinterpretation of others' intention) and to comprehend these typical errors by looking at individual experiences of the patient [99]. To overcome these typical cognitive errors associated with depressive or (hypo-)manic mood states strategies can be helpful that are also used in the treatment of depression as for example the Socratic dialogue or behavioral experiments and reality testing in order to reach cognitive restructuring.

A further component of the CBT for bipolar disorders is to keep a mood diary during the therapy. This is a good opportunity to collect information and to develop the ability to differentiate between normal and harmless mood swings or fluctuations in the sleeping pattern and first symptoms that are due to the illness [100]. With the aid of a better ability to differentiate between pathological and normal changes in mood and sleeping pattern the patient is able to recognize potential triggers and first symptoms of new episodes in time and to make use of promising coping strategies [101-102]. Coping strategies for depressive mood states comprise for example behavioral activation whereas the strategies of stimulus control and anti-impulsiveness are useful in (hypo-)manic mood states. It can be summarized that the main goal of CBT for bipolar disorder is to make the patients specialists concerning their own illness and to enable them to behave in a way that prevents the occurrence of prodromal symptoms or to counteract occurring prodromal symptoms in time.

In some cases it is also necessary to involve family members in the therapy of bipolar patients. Family members may for example be helpful to identify early warning symptoms as some patients are not able to identify them all alone. Furthermore, in some cases it needs to be discussed how family members can give feedback and offer help to the patient if they have the impression than the patient is developing a new affective episode. On the other hand it is often necessary to clarify and to illustrate that not all mood changes are necessarily indicators for the beginning of a further affective episode but are normal to a certain extent. 
As in the case of unipolar depression it is indispensable to address the issue of probable future crises during the last therapy sessions and to reflect possibilities that may help the patient to get along self-directed. Concrete emergency schedules should be developed for depressive symptoms as well as for (hypo-)manic symptoms. Furthermore, it is helpful to fade out therapeutic contacts slowly - just as in unipolar depression.

\section{Effectiveness and efficacy of cognitive behavioral therapy in the treatment of bipolar disorders}

During the last twenty years randomized controlled trials have shown that CBT for bipolar disorders is not only helpful to encourage medication adherence [93] but as an adjunct to medication also to reduce symptom severity, to accelerate stabilization, to delay relapses, and to improve the level of functioning [e.g., 103, 104-105]. In a recent systematic review Miklowitz and Scott included all randomized trials of adjunctive therapy for bipolar disorders that have been published between 1980 and 2008 [106]. While many of these studies examined the effectiveness of other psychosocial interventions (e.g., psychoeducation, systematic care management, family focused therapy) more than ten studies that have been included have specifically examined the effectiveness and efficacy of CBT for bipolar disorders. Even though Miklowitz and Scott point out that all these studies differ concerning many methodological issues (e.g., different kinds of CBT interventions, different sample characteristics) a great portion of these studies revealed that CBT is superior to routine care (i.e., mood stabilizers plus outpatient support) with respect to many outcome variables as for example the number of relapses, the improvement of social adjustment and the improvement of coping strategies to manage prodromal symptoms. Furthermore, patients who receive CBT seem to have less psychiatric admissions or days in an affective episode. This seems to be not only the case during the time of treatment but has also been shown for longer follow-up periods (e.g., 30 months). Interestingly, over a 30month follow-up CBT has not only been superior to TAU concerning the clinical outcome variables but also concerning the costs that have been associated with the respective treatment form.

However, most studies that have been conducted to examine the efficacy of CBT for bipolar disorders have compared the effects of CBT against the effects of a TAU condition. Thereby, in most cases the CBT group received the CBT in addition to what has been referred to as TAU (e.g., medication which is associated with regular visits to the psychiatrist). Hence, the patients allocated to the CBT group received more attention and support than patients who have been allocated to the control group in these studies. To control for the effects of the surplus in attention and support that has been associated with the CBT groups in former studies Meyer and Hautzinger conducted a randomized controlled trial in which they compared CBT to a supportive therapy (ST) [107]. The authors found no differences between the two treatment conditions with respect to relapse rates. Thus, it can be assumed that certain characteristics that are shared by many psychosocial interventions as for example providing information or systematic mood monitoring might explain the effects of different kinds of treatments for bipolar disorders. This finding fits to a meta-analysis conducted by 
Lynch and colleagues in which amongst others the effectiveness of CBT for bipolar disorders has been examined by pooling data from published trials of CBT that used controls for non-specific effects of intervention [108]. Furthermore, trials of effectiveness against relapse were also pooled if they compared CBT to TAU. In this analysis CBT proved to be ineffective in reducing relapse in bipolar disorder. The authors conclude that based on the present data CBT does not seem to be an effective treatment strategy for relapseprevention in bipolar disorder. Based on these findings Meyer and Hautzinger summarize that there is not much evidence for specific effects of CBT for bipolar disorders. Therefore, they suggest that future research should focus on matching patients to treatments, that is on examining possible moderator variables of CBT for bipolar disorders.

\section{Mediators and moderators of treatment effects of CBT for bipolar disorder}

While there are many studies dealing with moderators and mediators of CBT for depression studies examining the moderators and mediating mechanisms of CBT for bipolar disorders have rarely been conducted. However, there are first hints that patients with specific characteristics seem to benefit less respectively more from CBT than do other patients.

Some patient characteristics with unclear status: Prognostic factors or moderator variables for the response to CBT for bipolar disorders?

Lam et al. randomized over one hundred patients to either CBT or a control group and examined whether patients who score highly on the so-called Sense of Hyper-Positive Self Scale (SHPSS) do respond to CBT as well as other patients do [109]. The SHPSS measures to what extent patients suffering from a bipolar disorder value themselves and assume that they possess personal attributes that are associated with a state of being 'mildly high' (e.g., dynamism, productiveness). As expected the authors found that patients with high scores on the SHPSS had a significantly higher rate of relapse after controlling for other clinical variables that are relevant in this context. Another variable that seems to play a role in the responsiveness to CBT is the level of activation the patients display: In this context it has been revealed that patients who show heightened and more volatile activation scale scores show a worse response to CBT than patients who show a contrary pattern [110]. Furthermore, it seems as if patients with fewer than twelve previous affective episodes benefit more from CBT with regard to relapse that patients with more affective episodes [111].

The mediating role of dysfunctional attitudes and further potential mediators

Ball and colleagues conducted a randomized controlled trial of schema-focused CBT that made, besides the typical CBT techniques, use of emotive therapy techniques as for example imagery or reliving of early experiences [112]. In this study fifty-two bipolar patients have been randomized to either CBT plus mood stabilizers or treatment as usual (TAU) plus mood stabilizers. It has not only been revealed that patients who received CBT had lower depression scores at posttreatment but also that they displayed less dysfunctional attitudes than patients who were allocated to TAU. Furthermore, patients who received CBT seemed 
to show a greater time to depressive relapse as compared to the TAU patients (however, statistically seen this was only significant on a trend level). In the one-year follow-up CBT patients showed a trend toward lower manic symptoms and improved behavioral selfcontrol. Moreover, the change of the severity of illness status from baseline to 18-months follow-up changed more within the CBT group as compared to the TAU group. Even though some methodological issues can be brought up against this study (for example it remains unclear if schema-focused CBT has any advantages in the treatment of bipolar disorders as compared to 'pure' CBT) this study suggests that one mediating mechanism of CBT for bipolar disorder may lay in the change of dysfunctional attitudes. This suggestion finds support in a study by Zaretsky and colleagues in which seventy-nine patients suffering from bipolar disorder have been randomized to either psychoeducation plus CBT or a short course of psychoeducation (PE) alone [113]. While the groups did not differ concerning hospitalization rates, medication adherence, psychosocial functioning, or mental health use, it has been shown that patients who received CBT in addition to PE experienced $50 \%$ fewer days of depressed mood over the course of one year and needed antidepressant increases less frequently than patients allocated to PE. Furthermore, dysfunctional attitudes decreased significantly more in the CBT group than in the PE group, suggesting that this indeed might be a mediating variable. Another variable that has also been shown to change during CBT is the coping with prodromal symptoms [114]. Thus, it could be assumed that this might also be a mediating variable for the effectiveness of CBT. However, this association has never been specifically examined.

In contrast to the field of unipolar depression there are, to our knowledge, no studies concerning neural correlates of treatment response in bipolar disorders. Thus, one can only speculate that prefrontal and subcortical areas and networks that are involved in emotion regulation might play a mediating role in CBT for bipolar disorders.

Due to the lack of studies concerning moderators and mediators of CBT for bipolar disorders Miklowitz and Scott list a number of variables that "could provide viable explanations for the effectiveness of various forms of psychotherapy" [106]. Amongst other variables they list for example acquiring emotional self-regulation skills, improving social skills, enhancing medication adherence, stabilizing sleep/wake cycles and other daily routines, and improving the ability to identify and intervene early with relapses.

\section{New developments in the CBT for bipolar disorders}

While there is a growing body of research concerning cCBT for depression these new developments have hardly been used in the treatment of bipolar disorders. In 2007 Barnes and colleagues described an internet-based disease management platform that has been developed by Sentiens, a mental health service provider in Perth (Australia), called 'Recovery Road' (RR) [115-116]. This platform enables patients and their treating clinicians to keep contact by the use of asynchronous messaging. Furthermore, patients and clinicians are provided with self-report monitoring tools including different online mental health, medication adherence, and quality-of-life questionnaires so that they receive immediate 
feedback on progress, medication adherence and risk of relapse. Moreover, users have access to a one-year relapse prevention program for bipolar disorders (or psychosocial treatments for other mental illnesses) including ten sessions of CBT that has been developed by Barnes. As far as we know, the results of the randomized controlled trial that has been conducted by Barnes and colleagues to evaluate this CCBT program for bipolar disorder have not been published so far. Therefore, it has to be awaited if CCBT can also be helpful in the treatment of bipolar disorder.

\section{Gaps of knowledge and future directions}

As we have seen compared to the field of bipolar disorders there are many studies examining potential moderator and mediator variables of CBT for depression. However, in some cases there are contradictory findings that do not allow drawing definite conclusions. For example it is still not clear whether more severe and complicated patients do better or worse in CBT treatment as compared to patients with a less severe symptomatic. It may be that the heterogeneity of the findings concerning this specific patient characteristic is at least in part due to a different extent of therapists' expertise. Therefore, in future studies concerning the outcome of CBT in depression, we recommend to control for the expertise status of therapists, to elucidate the role of illness severity and comorbid personality disorders. In this context it seems important to mention a study that has been conducted by Strunk and colleagues [117]. In this study the therapists' competence has been rated and the authors found that the adequacy of therapists' delivery of the treatment is predictive for symptom change early in treatment. Furthermore, Strunk et al. investigated whether therapists' competence is more important to patients with specific complicating features and found that competence seems to be more important to patients who were characterized by higher anxiety, an earlier age of onset, and who suffered from a chronic form of depression as compared to patients without these characteristics. However, patients with a comorbid personality disorder did not have a greater benefit in dependence of the therapists' competence as compared to those patients who didn't meet criteria for a personality disorder. This study impressively illustrates that the expertise of the therapists may have a strong influence on the findings concerning potential moderator variables of CBT. Therefore, it needs to be taken into account in future studies.

An even more far-reaching problem in the context of studies searching for potential moderator variables of CBT for depression is that in many cases it is not strictly distinguished between moderator variables on one side and prognostic factors on the other. Thus, many studies only examine whether a variable predicts the treatment response to CBT whereas studies also including other psychosocial interventions in addition to CBT to examine the differential prognostic value of potential moderator variables are rather limited. Therefore, it seems necessary to disentangle the meaning of 'predictors/prognostic factors' versus 'moderator variables' and to clearly carve out whether a variable is one or the other. For example, based on findings that demonstrate that those patients benefit most from CBT who show an increased emotional reactivity pretreatment and who are not able to engage regulatory structures pretreatment, Siegle and colleagues propose that the presence of 
emotion regulation deficits, which are targeted in CBT, may be the key to recovery with this specific intervention. Even though this assumption seems plausible, up till now it is not clear whether this prediction is specific to CBT. It is also possible that the same pattern could be found for other psychosocial interventions, as for example for IPT. To be able to decide what treatment to select for which patient it is necessary to go beyond the investigation of prognostic factors and to examine moderating variables.

A structurally related argument can be brought up against some studies investigating mediator variables. Strictly speaking, some studies that examine mediating mechanisms do not clearly distinguish variables mediating the response to CBT or variables being part of improvement rather than being a cause of improvement. To disentangle this more clearly, it would be required to assess the mediating as well as the outcome variable not only at study entry and the end of the study but also repeatedly during the therapy to make sure that the change of the mediator variable really precedes the change of the outcome variable and to rule out reverse causality.

While more and especially more carefully designed studies are already required concerning moderating and mediating variables of face-to-face CBT this is even more the case for the relatively new developments of $\mathrm{CCBT}$ and $\mathrm{tCBT}$. Future studies should shed light on the question if CCBT and $\mathrm{tCBT}$ might be helpful especially for those patients who do not benefit as much from traditional CBT as do other patients. If so, these new developments could be routinely implemented into the health care systems and be provided especially to those patients who are not addressed successfully so far. The examination of the therapeutic usefulness of technical devices such as smart phones, tablet pc and apps will be another area of future research.

Even though there are some promising results concerning the effectiveness and efficacy of CBT for bipolar disorders until now it is not clear whether these findings can be traced back to active factors that are specific to CBT or whether these are due to rather unspecific factors that are associated with any kind of psychosocial treatment and even with supportive interventions. Furthermore, to date there is hardly any knowledge concerning moderator variables of the response to CBT for bipolar disorders and concerning mediating mechanisms. However, knowing moderator variables means to have a basis for choosing the best treatment for a given patient and thus is indispensable to improve our treatment assignments as well as to reduce our health care costs. Therefore, it seems necessary to broaden our knowledge in this context. The same holds true for mediating variables as these provide us with an answer to the question how CBT works and thus not only allow to identify mechanisms of change within the patient but also to identify active therapy ingredients in the treatment process. This in turn can be used to develop even more efficacious forms of therapy.

\section{Author details}

Larissa Wolkenstein and Martin Hautzinger

University of Tübingen, Germany 


\section{References}

[1] Hautzinger, M., Depression. Fortschritte der Psychotherapie. Manuale für die Praxis, ed. D. Schulte, et al. 1998, Göttingen: Hogrefe.

[2] Beck, A.T., et al., Cognitive therapy of depression. 1979, New York: Guilford Press.

[3] Elkin, I., et al., National Institute of Mental Health Treatment of Depression Collaborative Research Program: general effectiveness of treatments. Archives of General Psychiatry, 1989. 46: p. 971-982.

[4] DeRubeis, R.J., et al., Cognitive therapy vs. medications in the treatment of moderate to severe depression. Archives of General Psychiatry, 2005. 62: p. 409-416.

[5] Casacalenda, N., J.C. Perry, and K. Looper, Remission in major depressive disorder: a comparison of pharmacotherapy, psychotherapy, and control conditions. American Journal of Psychiatry, 2002. 159: p. 1354-1360.

[6] Hollon, S.D., M.E. Thase, and J.C. Markowitz, Treatment and prevention of depression. Psychological Science in the Public Interest, 2002. 3: p. 1-39.

[7] Elkin, I., et al., Initial severity and differential treatment outcome in the National Institute of Mental Health Treatment of Depression Collaborative Research Program. Journal of Consulting and Clinical Psychology, 1995. 63: p. 841-847.

[8] American Psychiatric Association, Practice guideline for the treatment of patients with major depressive disorder (revision). American Journal of Psychiatry, 2000. 157: p. 1-45.

[9] DeRubeis, R.J., et al., Medications versus cognitive behavior therapy for severely depressed outpatients: mega-analysis of four randomized comparisons. American Journal of Psychiatry, 1999. 156: p. 1007-1013.

[10] Hollon, S.D., Cognitive and behavior therapy in the treatment and prevention of depression. Depression and Anxiety, 2011. 28: p. 263-266.

[11] Hollon, S.D., et al., Psychotherapy and medication in the treatment of adult and geriatric depression: which monotherapy or combined treatment? Journal of Clinical Psychiatry, 2005. 66: p. 455-468.

[12] Hollon, S.D., M.O. Stewart, and D. Strunk, Cognitive behavior therapy has enduring effects in the treatment of depression and anxiety. Annual Review of Psychology, 2006. 57: p. 285315.

[13] Hollon, S.D., et al., Prevention of relapse following cognitive therapy vs medications in moderate to severe depression. Archives of General Psychiatry, 2005. 62: p. 417-422.

[14] Evans, M.D., et al., Differential relapse following cognitive therapy and pharmacotherapy for depression. Archives of General Psychiatry, 1992. 49: p. 802-808.

[15] Frank, E., et al., Three-year outcomes for maintenance therapies in recurrent depression. Archives of General Psychiatry, 1990. 47: p. 1093-1099.

[16] Jarrett, R.B., et al., Preventing Recurrent Depression Using Cognitive Therapy With and Without a Continuation Phase. A Randomized Clinical Trial. Archives of General Psychiatry, 2001. 58: p. 381-388.

[17] Teasdale, J.D., et al., Prevention of Relapse/Recurrence in Major Depression by MindfulnessBased Cognitive Therapy. Journal of Consulting and Clinical Psychology, 2000. 68: p. 615623. 
[18] Scott, J., et al., Effects of cognitive therapy on psychosocial symptoms and social functioning in residual depression. British Journal of Psychiatry, 2000. 177: p. 440-446.

[19] Paykel, E., et al., Prevention of relapse by cognitive therapy in residual depression: a controlled trial. Archives of General Psychiatry, 1999. 56: p. 829-835.

[20] Jacobson, N.S., et al., A component analysis of cognitive-behavioral treatment for depression. Journal of Consulting and Clinical Psychology, 1996(64): p. 295-304.

[21] Dimidjian, S., et al., Randomized Trial of Behavioral Activation, Cognitive Therapy, and Antidepressant Medication in the Acute Treatment of Adults With Major Depression. Journal of Consulting and Clinical Psychology, 2006. 74: p. 658-670.

[22] Power, M.J. and C. Freeman, A randomized controlled trial of IPT versus CBT in primary care: With some cautionary notes about handling missing values in clinical trials. Clinical Psychology and Psychotherapy, 2012. 19: p. 159-169.

[23] Luty, S.E., et al., Randomised controlled trial of interpersonal psychotherapy and cognitivebehavioural therapy for depression. The British Journal of Psychiatry, 2007. 190(6): p. 496502.

[24] de Mello, M.F., et al., A systematic review of research findings on the efficacy of interpersonal therapy for depressive disorders. European Archives of Psychiatry and Clinical Neuroscience, 2005(255): p. 75-82.

[25] Cuijpers, P., et al., Depression and dysthymic disorders, in Handbook of evidence-based practice in clinical psychology, M. Hersen and P. Sturmey, Editors. 2012, Wiley.

[26] Cuijpers, P., et al., Psychotherapy for depression in adults: A meta-analysis of comparative outcome studies. Journal of Consulting and Clinical Psychology, 2008. 76: p. 909-922.

[27] Cuijpers, P., A. van Straten, and L. Warmerdam, Are individual and group treatments equally effective in the treatment of depression in adults? A meta-analysis. European Journal of Psychiatry, 2008. 22: p. 38-51.

[28] Cuijpers, P., et al., The effects of psychotherapy for adult depression are overestimated: A metaanalysis of study quality and effect size. Psychological Medicine, 2010. 40: p. 211-223.

[29] Thoma, N.C., et al., A quality-based review of randomized controlled trials of cognitivebehavioral therapy for depression: An assessment and metaregression. American Journal of Psychiatry, 2012. 169: p. 22-30.

[30] Cuijpers, P., et al., Self-reported versus clinician-rated symptoms of depression as outcome measures in psychotherapy research on depression: A meta-analysis. Clinical Psychology Review, 2010. 30: p. 768-778.

[31] Antonuccio, D.O., M. Thomas, and W.G. Danton, A cost-effectiveness analysis of cognitive behavior therapy and fluoxetine (Prozac) in the treatment of depression. Behavior Therapy, 1997. 28: p. 187-210.

[32] Brown, J.S.L., et al., Outcome, costs and patient engagement for group and individual CBT for depression: A naturalistic clinical study. Behavioural and Cognitive Psychotherapy, 2011. 39: p. 355-358.

[33] Kraemer, H.C., et al., Mediators and moderators of treatment effects in ranomized clinical trials. Archives of General Psychiatry, 2002. 59: p. 877-883. 
[34] Sotsky, S.M., et al., Patient predictors of response to psychotherapy and pharmacotherapy: Findings in the NIMH Treatment of Depression Collaborative Research Program. American Journal of Psychiatry, 1991. 148: p. 997-1008.

[35] Carter, J.D., et al., Patient predictors of response to cognitive behaviour therapy and interpersonal psychotherapy in a randomized clinical trial for depression. Journal of Affective Disorders, 2011. 128: p. 252-261.

[36] Fournier, J.C., et al., Prediction of response to medication and cognitive therapy in the treatment of moderate to severe depression. Journal of Consulting and Clinical Psychology, 2009. 77: p. 775-787.

[37] Thase, M.E., et al., Relapse after cognitive behavior therapy of depression - potential implications for longer courses of treatment. American Journal of Psychiatry, 1992. 149: p. 1046-1052.

[38] Jarrett, R.B., et al., Clinical, cognitive, and demographic-predictors of response to cognitive therapy for depression - a preliminary-report. Psychiatry Research, 1991. 37: p. 245-260.

[39] Safran, J.D., et al., Assessing patient suitability for short-term cognitive therapy with interpersonal focus. Cognitive Therapy and Research, 1993. 17: p. 23-38.

[40] Young, J.E., Cognitive therapy for personality disorders: A schema-focused approach. 1990, Sarasota, FL: Professional Resource Exchange Inc.

[41] Halford, W.K., S. Nernoth-Doolan, and K. Eadie, Schemata as moderators of clinical effectiveness of a comprehensive cognitive behavioral program for patients with depression or anxiety disorder. Behavior Modification, 2002. 26: p. 571-593.

[42] Teismann, T., et al., Bedeutung von Rumination und Ablenkung für den Therapieerfolg depressiver Patienten. Verhaltenstherapie, 2008. 18: p. 215-222.

[43] Teismann, T., et al., Influence of rumination and distraction on the therapeutic process in cognitive-behavioral therapy for depression. Cognitive Therapy and Research, 2012. 36: p. 15-24.

[44] Arnow, B.A., et al., Rumination and distraction among chronic depressives in treatment: A structural equation analysis. Cognitive Therapy and Research, 2004. 28: p. 67-83.

[45] Siegle, G.J., et al., Do the seconds turn into hours? Relationships between sustained pupil dilation in response to emotional information and self reported rumination. Cognitive Therapy and Research, 2003. 27: p. 365-383.

[46] Siegle, G.J., et al., Can't shake that feeling: fMRI assessment of sustained amygdala activity in response to emotional information in depressed individuals. Biological Psychiatry, 2002. 51: p. 693-707.

[47] Deldin, P.J., A.Y. Shestyuk, and P.H. Chiu, Event-related brain potential indices of memory biases in major depression, in Principles of experimental psychopathology: Essays in honor of Brendan A. Maher, M.F. Lenzenweger and J.M. Hooley, Editors. 2003, American Psychological Association. p. 195-209.

[48] Sheline, Y.I., et al., Increased amygdala response to masked emotional faces in depressed subjects resolves with antidepressant treatment: an fMRI study. Biological Psychiatry, 2001. 50: p. 651-658. 
[49] Siegle, G.J., C.S. Carter, and M.E. Thase, Use of fMRI to predict recovery from unipolar depression with cognitive behavior therapy. American Journal of Psychiatry, 2006. 163: p. 735-738.

[50] Fournier, J.C., et al., Antidepressant medications v. cognitive therapy in people with depression with or without personality disorder. British Journal of Psychiatry, 2008. 192: p. 124-129.

[51] Coffman, S.J., et al., Extreme nonresponse in cognitive therapy: can behavioral activation succeed where cognitive therapy fails. Journal of Consulting and Clinical Psychology, 2007. 75: p. 531-541.

[52] Barber, J.P. and L.R. Muenz, The role of avoidance and obsessiveness in matching patients to cognitive and interpersonal psychotherapy: Empirical findings from the treatment of Depression Collaborative Research Program. Journal of Consulting and Clinical Psychology, 1996. 64: p. 951-958.

[53] Stangier, U., et al., Maintenance Cognitive Therapy and Manualized Psychoeducation in the Treatment of Recurrent Depression: A Prospective Randomized Multicenter Controlled Trial. American Journal of Psychiatry, in press.

[54] McBride, C., et al., Attachment as moderator of treatment outcome in major depression: A randomized control trial of interpersonal psychotherapy versus cognitive behavior therapy. Journal of Consulting and Clinical Psychology, 2006. 74: p. 1041-1054.

[55] Joyce, P.R., et al., Temperament, character and personality disorders as predictors of response to interpersonal psychotherapy and cognitive-behavioral therapy for depression. British Journal of Psychiatry, 2007. 190: p. 503-508.

[56] Kocsis, J.H., et al., Journal of Clinical Psychiatry. Patient preference as a moderator of outcome for chronic forms of major depressive disorder treated with nefazodone, cognitive behavioral analysis system of psychotherapy, or the combination, 2009. 70: p. 354-361.

[57] Leykin, Y., et al., The relation of patients' treatment preference to outcome in a randomized clinical trial. Behavioral Therapy, 2007. 38: p. 209-217.

[58] Goldapple, K., et al., Modulation of cortical-limbic pathways in major depression: treatmentspecific effects of cognitive behavior therapy. Archives of General Psychiatry, 2004. 61: p. 3441.

[59] Mayberg, H., et al., Regional metabolic effects of fluoxetine in major depression: Serial changes and relationship to clinical response. Biological Psychiatry, 2000. 48: p. 830-843.

[60] Fu, C.H.Y., et al., Attenuation of the neural response to sad faces in major depression by antidepressant treatment: a prospective, event-related functional magnetic resonance imaging study. Archives of General Psychiatry, 2004. 61: p. 877-889.

[61] DeRubeis, R.J., G.J. Siegle, and S.D. Hollon, Cognitive therapy vs. medications for depression: Treatment outcomes and neural mechanisms. Nature Reviews Neuroscience, 2008. 9: p. 788-796.

[62] Kennedy, S.H., et al., Differences in brain glucose metabolism between responders to CBT and venlafaxine in a 16-week randomized controlled trial. American Journal of Psychiatry, 2007. 164: p. 778-788. 
[63] Fu, C.H.Y., et al., Neural Responses to Sad Facial Expressions in Major Depression Following Cognitive Behavioral Therapy. Biological Psychiatry, 2008. 64: p. 505-512.

[64] Ritchey, M., et al., Neural correlates of emotional processing in depression: Changes with cognitive behavioral therapy and predictors of treatment response. Journal of Psychiatric Research, 2011. 45: p. 577-587.

[65] Beck, A.T., Cognitive therapy: A 30-year retrospective. American Psychologist, 1991. 46: p. 368-375.

[66] Manicavasagar, V., T. Perich, and G. Parker, Cognitive predictors of change in cognitive behaviour therapy and mindfulness-based cognitive therapy for depression. Behavioural and Cognitive Psychotherapy, 2012. 40: p. 227-232.

[67] Imber, S.D., et al., Mode-specific effects among three treatments for depression. Journal of Consulting and Clinical Psychology, 1990. 5: p. 352-359.

[68] Simons, A.D., S.L. Garfield, and G.E. Murphy, The process of change in cognitive therapy and pharmacotherapy for depression. Archives of General Psychiatry, 1984. 41: p. 45-51.

[69] Oei, T.P.S. and M.L. Free, Do cognitive behaviour therapies validate cognitive models of mood disorder? A review of the empirical evidence. International Journal of Psychology, 1995. 30: p. $145-179$.

[70] DeRubeis, R.J., et al., How does cognitive therapy work? Cognitive change and symptom change in cognitive therapy and pharmacotherapy for depression. Journal of Consulting and Clinical Psychology, 1990. 58: p. 862-869.

[71] Strunk, D.R., et al., Patients' competence in and performance of cognitive therapy skills: relation to the reduction of relapse risk following treatment for depression. Journal of Consulting and Clinical Psychology, 2007. 75: p. 523-530.

[72] Segal, Z.V., et al., Cognitive reactivity to sad mood provocation and the prediction of depressive relapse. Archives of General Psychiatry, 2006. 63: p. 749-755.

[73] Hollon, S.D., M.D. Evans, and R.J. DeRubeis, Cognitive mediation of relapse prevention following treatment for depression: Implications of differential risk, in Psychological Aspects of Depression, R.E. Ingram, Editor. 1990, Plenum: New York. p. 117-136.

[74] Teasdale, J.D., et al., How does cognitive therapy prevent relapse in residual depression: evidence from a controlled trial. Journal of Consulting and Clinical Psychology, 2001. 69: p. 347-357.

[75] Hollon, S.D., et al., Prevention of relapse following cognitive therapy versus medications in moderate to severe depression. Archives of General Psychiatry, 2005. 62: p. 417-422.

[76] Tang, T.Z. and R.J. DeRubeis, Sudden gains and critcal sessions in cognitive-behavioral therapy for depression. Journal of Consulting and Clinical Psychology, 1999. 67: p. 894904.

[77] Keller, F. and M. Hautzinger, Klassifikation von Verlaufskurven in der Depressionsbehandlung. Zeitschrift für Klinische Psychologie und Psychotherapie, 2007. 36: p. 83-92.

[78] Foroushani, P.S., J. Schneider, and N. Assareh, Meta-review of the effectiveness of computerised CBT in treating depression. BMC Psychiatry, 2011. 11: p. 131-136. 
[79] Horvitz-Lennon, M., et al., "Usual care" for major depression in the 1990s: Characteristics and expert-estimated outcomes. American Journal of Psychiatry, 2003. 160: p. 720-726.

[80] Wierzbicki, M. and G. Pekarik, A metaanalysis of psychotherapy dropout. Professional Psychology: Research and Practice, 1993. 24: p. 190-195.

[81] Griffiths, K.M., L. Farrer, and H. Christensen, The efficacy of internet interventions for depression and anxiety disorders: a review of randomised controlled trials. 2010. 192: p. 4-11.

[82] Andersson, G. and P. Cuijpers, Internet-based and other computerized psychological treatments for adult depression: A meta-analysis. Cognitive Behaviour Therapy, 2009. 38: p. 196-205.

[83] Spek, V., et al., Internet-based cognitive behaviour therapy for symptoms of depression and anxiety: A meta-analysis. Psychological Medicine, 2007. 37: p. 319-328.

[84] Lynch, D., M. Tamburrino, and R. Nagel, Telephone counseling for patients with minor depression: Preliminary findings in a family practice setting. Journal of Family Practice, 1997. 44: p. 293-298.

[85] Mohr, D.C., et al., Telephone-administered psychotherapy for depression. Archives of General Psychiatry, 2005. 65: p. 1007-1014.

[86] Mohr, D.C., et al., Effect of telephone-administered vs face-to-face cognitive behavioral therapy on adherence to therapy and depression outcomes among primary care patients: a randomized trial. The Journal of the American Medical Association, 2012. 307: p. 2278-2285.

[87] Ludman, E.J., et al., A randomized trial of telephone psychotherapy and pharmacotherapy for depression: Continuation and durability effects. Journal of Consulting and Clinical Psychology, 2007. 75: p. 257-266.

[88] Button, K.S., et al., Factors associated with differential response to online cognitive behavioural therapy. Social Psychiatry and Psychiatric Epidemiology, 2012. 47: p. 827-833.

[89] Kessler, D., et al., Therapist-delivered internet psychotherapy for depression in primary care: $a$ randomized controlled trial. Lancet, 2009. 374: p. 628-634.

[90] Wiersma, J.E., et al., Treating chronic symptoms of depression in the virtual clinic: Findings on chronicity of depression in patients treated with internet-based computerized cognitive behaviour therapy for depression. Psychotherapy and Psychosomatics, 2011. 80: p. 313-315.

[91] de Graaf, L.E., S.D. Hollon, and M.J. Huibers, Predicting outcome in computerized cognitive behavioral therapy for depression in primary care: A randomized trial. Journal of Consulting and Clinical Psychology, 2010. 78: p. 184-189.

[92] Warmerdam, L., et al., Online cognitive behavioral therapy and problem-solving therapy for depressive symptoms: Exploring mechanisms of change. Journal of Behavior Therapy and Experimental Psychiatry, 2010. 41: p. 64-70.

[93] Cochran, S.D., Preventing medical noncompliance in the outpatient treatment of bipolar disorders. Journal of Consulting and Clinical Psychology, 1984. 52: p. 873-878.

[94] Alloy, L.B., et al., The psychosocial context of bipolar disorder: environmental, cognitive, and developmental risk factors. Clinical Psychology Review, 2005. 25: p. 1043-1075.

[95] Johnson, S.L., Mania and dysregulation in goal pursuit: a review. Clinical Psychology Review, 2005. 25: p. 241-262. 
[96] Basco, M.R. and A.J. Rush, Cognitive-behavioral therapy for bipolar disorder. 1996, New York: Guilford.

[97] Lam, D., et al., Cognitive therapy for bipolar disorder. A therapist's guide to concepts, methods and practice. Vol. 2. 2010, New York: Wiley.

[98] Meyer, T.D. and M. Hautzinger, Manisch depressive Störungen - Kognitive Verhaltenstherapie zur Rückfallprophylaxe. 2004, Weinheim: Beltz.

[99] Hautzinger, M. and T.D. Meyer, Bipolare affektive Störungen. Fortschritte der Psychotherapie. 2011, Göttingen: Hogrefe.

[100] Meyer, T.D., Bipolare Störungen, in Lehrbuch der Verhaltenstherapie, J. Margraf and S. Schneider, Editors. 2008, Springer: Berlin.

[101] Bauer, M., et al., Using technology to improve longitudinal studies: self-reporting with ChronoRecord in bipolar disorder. Bipolar Disorders, 2004. 6: p. 67-74.

[102] Lam, D. and G. Wong, Prodromes, coping strategies and psychological interventions in bipolar disorders. Clinical Psychology Review, 2005. 25.

[103] Miklowitz, D.J., Adjunctive psychotherapy for bipolar disorder: state of evidence. American Journal of Psychiatry, 2008. 165: p. 1408-1419.

[104] Scott, J., Psychotherapy for bipolar disorders - efficacy and effectiveness. Journal of Psychopharmacology, 2006. 20: p. 46-50.

[105] Lam, D.H., et al., Cognitive therapy for bipolar illness: pilot study of relapse prevention. Cognitive Therapy and Research, 2000. 24: p. 503-520.

[106] Miklowitz, D.J. and J. Scott, Psychosocial treatments for bipolar disorder: cost-effectiveness, mediating mechanisms, and future directions. Bipolar Disorders, 2009. 11: p. 110-122.

[107] Meyer, T.D. and M. Hautzinger, Cognitive behaviour therapy and supportive therapy for bipolar disorders: relapse rates for treatment period and 2-year follow-up. Psychological Medicine, 2012. 42: p. 1429-1439.

[108] Lynch, D., K.R. Laws, and P.J. McKenna, Cognitive behavioural therapy for major psychiatric disorder: does it really work? A meta-analytical review of well-controlled trials. Psychological Medicine, 2010. 40: p. 9-24.

[109] Lam, D., K. Wright, and P. Sham, Sense of hyper-positive self and response to cognitive therapy in bipolar disorder. Psychological Medicine, 2005. 35: p. 69-77.

[110] Scott, J., A. Garland, and S. Moorhead, A pilot study of cognitive therapy in bipolar disorders. Psychological Medicine, 2001. 31: p. 459-467.

[111] Scott, J., et al., Cognitiv behaviour therapy for severe and recurrent bipolar disorders: a randomised controlled trial. British Journal of Psychiatry, 2006. 188: p. 313-320.

[112] Ball, J.R., et al., A randomized controlled trial of cognitive therapy for bipolar disorder: focus on long-term change. Journal of Clinical Psychiatry, 2006. 67: p. 277-286.

[113] Zaretsky, A., et al., Is cognitive-behavioural therapy more effective than psychoeducation in bipolar disorder? Canadian Journal of Psychiatry, 2008. 53: p. 441-448.

[114] Lam, D.H., et al., A randomized controlled study of cognitive therapy for relapse prevention for bipolar affective disorder: outcome of the first year. Archives of General Psychiatry, 2003. 60: p. 145-152. 
[115] Tannenbaum, D. and C. Barnes, Infrapsych bipolar affective disorder recovery road program. Bipolar Disorders, 2004. 6: p. 24.

[116] Barnes, C., et al., Evaluation of an Online Relapse Prevention Program for Bipolar Disorder. An Overview of the Aims and Methodology of a Randomized Controlled Trial. Disease Management and Health Outcomes, 2007. 15: p. 215-224.

[117] Strunk, D.R., et al., Therapist Competence in Cognitive Therapy for Depression: Predicting Subsequent Symptom Change. Journal of Consulting and Clinical Psychology, 2010: p. 429-437. 